\title{
Kinematics of Star Clusters in M33: Distinct Populations
}

\author{
Rupali Chandar, Luciana Bianchi, Holland C. Ford \\ Johns Hopkins University, Department of Physics \& Astronomy, 3400 \\ N. Charles St., Baltimore, MD 21218 USA \\ \& Ata Sarajedini \\ Department of Astronomy, University of Florida, P. O. Box 112055, \\ Gainesville, FL 32611-2055 USA
}

\begin{abstract}
We analyse star cluster properties in the nearby spiral galaxy M33, combining our extensive HST WFPC2 photometry and groundbased follow up spectroscopy. These data show that cluster velocity dispersion increases with age. Simulations comparing synthetic disk and halo populations with observations indicate a composite disk/halo system for the old M33 clusters. The best fit fraction of $85 \pm 5 \%$ halo plus $15 \pm 5 \%$ disk differs from that found in the Milky Way, where $\sim 30 \%$ of the globular clusters are metal rich objects associated with the bulge and/or thick disk. Spectroscopic line indices for a halo subsample (17 objects) reveal an age spread of $\sim 5-7 \mathrm{Gyr}$, and little progression in metal abundance with age. This is consistent with a chaotic formation for a substantial portion of the M33 halo, and also consistent with an accretion origin for a large number of the halo clusters.
\end{abstract}

\section{Results}

Kinematic properties, age, and chemical composition are basic characteristics of stellar populations which provide powerful constraints on galaxy formation and evolution models. M33 is a nearby $(\sim 800 \mathrm{kpc})$, low luminosity, late-type (Scd) spiral galaxy, which is known to have formed a large system of massive star clusters over its lifetime (Christian \& Schommer 1988; Chandar, Bianchi, \& Ford 1999, hereafter CBF). To study the early formation of M33, we have used 45 multiband $H S T$ WFPC2 archive fields to detect clusters, and measure their luminosities, colors, and structural parameters (CBF 1999, 2001). Based on this sample, we obtained ground based integrated spectra for over 100 compact clusters with the WIYN 3.5-m telescope.

We compared velocity measurements with local disk motions as a function of cluster age (determined by comparing integrated colors with the single stellar population models of Bertelli et al. 1994), and found that the velocity dispersion dramatically increases with age (Figure 1-left). Schommer et al. (1991) determined from velocities of 14 red clusters in M33 that these comprise a distinct halo component, different from the young disk clusters. However with their small sample, they were unable to find any evidence for distinct subpopulations 

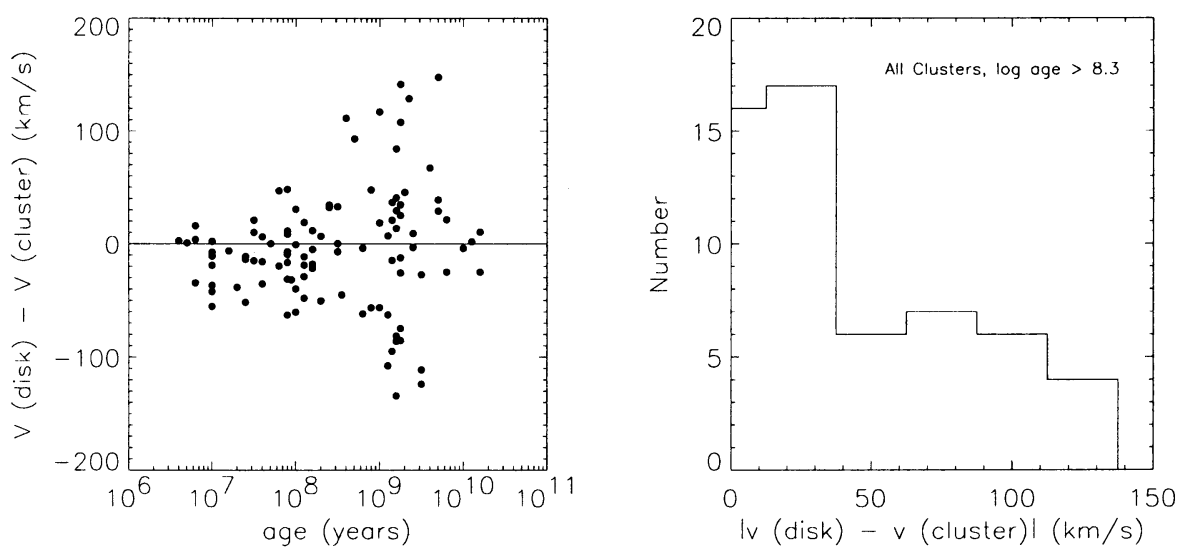

Figure 1. Left: $\left(V_{d i s k}-V_{c l}\right)$ as a function of age for clusters. Right: Histogram distribution of all clusters with (photometric) ages $\geq 8$.3yr in the log. This limit is where the velocity dispersion with age relation clearly appears to be maximized in Figure 1-left.

among the old clusters, as are known to exist in the Galaxy (e.g., Zinn 1985; Armandroff 1989) and M31 (e.g., Huchra et al. 1991). Because we have a larger sample of old objects, we used our velocity measurements to look for evidence of subpopulations (the sample is shown in Figure 1-right). Statistical simulations of pure (isotropic) "disk" and "halo" populations compared with our observed distribution show that it is unlikely that the old M33 clusters reside in a disk with velocity dispersion of $\sim 50 \mathrm{~km} \mathrm{~s}^{-1}$ or less (Chandar et al. 2002). When different fractions of simulated disk/halo clusters were compared with our observations, a fraction of $15 \pm 5 \%$ (thin) disk plus $85 \pm 15 \%$ halo gave a match at greater than $95 \%$ confidence, more than $90 \%$ of the time.

We then chose a subsample of 17 halo clusters in M33 that appear at the tails of the velocity histogram $\left(\left|V_{\text {disk }}-v_{\text {cluster }}\right| \geq 70 \mathrm{~km} \mathrm{~s}^{-1}\right)$ when cluster velocity is compared with local disk motion. These comprise a pristine halo sample. If their properties are representative of the overall M33 halo cluster population, we can constrain the early history of this galaxy. For our subsample of 17 halo clusters, we measured spectroscopic line indices as defined in Worthey et al. (1994) and used the Worthey (1994) models to derive ages and metal abundance. By comparing these M33 clusters with 12 Galactic globulars from Cohen et al. (1998), we find that these objects have an age spread $\sim 2$ times that of the Galactic GC sample, or $\sim 5-7$ Gyrs (Figure 2). Further, we find that most of these halo objects are relatively metal poor, even the intermediate age ones.

Based on the composite age and metallicity properties we conclude that: (i) $\approx 60 \%$ of our halo cluster subsample appears to be several Gyr younger than typical Galactic GCs, i.e., a much higher fraction of young to old halo clusters than found in the Milky Way and M31; (ii) the age-metallicity relationship of our halo sample, supported by the lack of an abundance gradient found by 
Sarajedini et al. (2000) for 10 old M33 clusters, is consistent with an accretion or chaotic origin for a large fraction of the M33 cluster halo.
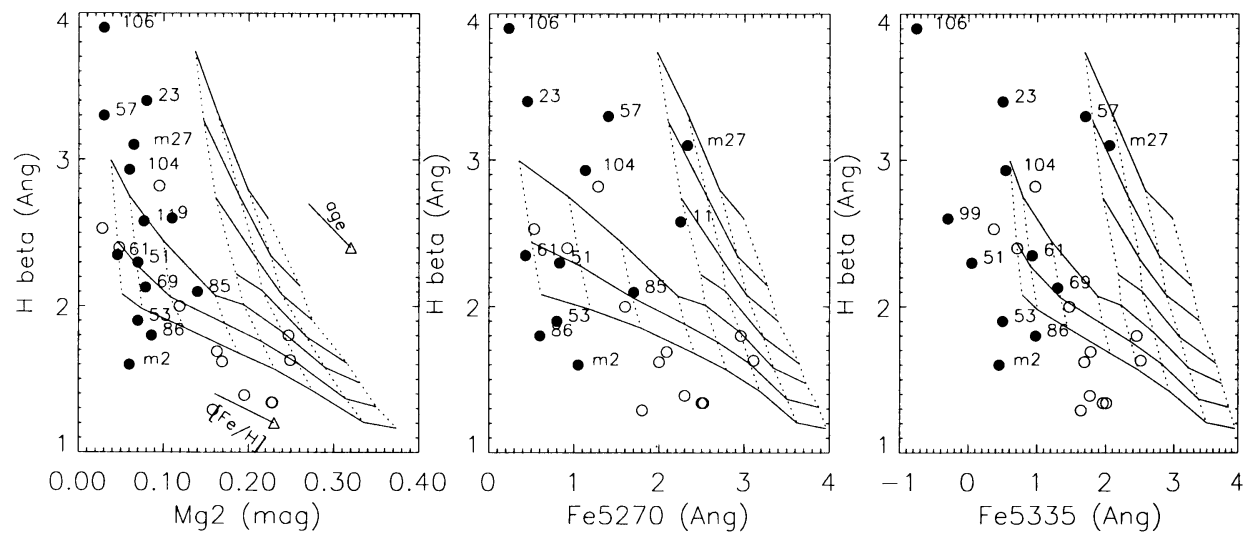

Figure 2. Spectroscopic line index measurements for a subsample of M33 halo clusters are shown as the solid circles. For comparison, line strength measurements of 12 Galactic globular clusters taken by Cohen et al. (1998) are shown as the open circles. Typical Balmer line uncertainties for our sample are $\pm 0.4-0.5$. The solid lines represent the Worthey (1994) models for ages of $1.5 \mathrm{Gyr}, 2 \mathrm{Gyr}, 3 \mathrm{Gyr}, 5 \mathrm{Gyr}$, $8 \mathrm{Gyr}, 12 \mathrm{Gyr}$, and $17 \mathrm{Gyr}$, with age increasing along the direction of the arrow. Abundances are represented by the short dashed lines, and for the $17 \mathrm{Gyr}, 12 \mathrm{Gyr}$ and $8 \mathrm{Gyr}$ model include $[\mathrm{Fe} / \mathrm{H}]$ values of -2.0 , $-1.5,-1.0,-0.5,-0.25,0.0,0.25,0.50$, and for the $1.5 \mathrm{Gyr}, 2 \mathrm{Gyr}, 3$ Gyr, and 5 Gyr models include $[\mathrm{Fe} / \mathrm{H}]$ values of $-0.22,0.0,0.25,0.50$, with metallicity increasing along the direction indicated.

\section{References}

Armandroff, T. E. 1989, AJ, 97, 375

Bertelli, G. et al. 1994, A \& AS, 106, 275

Chandar, R., Bianchi, L., \& Ford, H. C. 1999, ApJ, 517, 431

Chandar, R., Bianchi, L., \& Ford, H. C. 2001, A \& A, 366, 498

Chandar, R., Bianchi, L., Ford, H. C., \& Sarajedini, A. 2002, ApJ, 564, 712

Christian, C., \& Schommer, R. 1988, AJ, 95, 704

Cohen, J., Blakeslee, J., \& Ryhzov, A. 1998 ApJ, 496, 808

Huchra, J. P., Kent, S. M., \& Brodie, J. P. 1991, ApJ, 370, 495

Sarajedini, A., Geisler, D., Schommer, R., \& Harding, P. 2000, AJ, 120, 2437

Schommer, R. et al. 1991, AJ, 101, 873

Worthey, G. 1994, ApJS, 95, 107

Zinn, R. 1985, ApJ, 293, 424 